\title{
Combining Ability and Gene Action in Sesame (Sesamum indicum L.) Elite Genotypes by Half Diallel Mating Design
}

\author{
S. J. Sonawane*, P. B. Wadikar, M. R. Magar and S. L. Dhare \\ College of Agriculture, Latur, Vasantrao Naik Marathwada Krishi Vidyapeeth, \\ Parbhani - 431 402(MS), India \\ *Corresponding author
}

\section{Keywords \\ Combining ability, gene action, half diallel and sesame. \\ Article Info \\ Accepted: \\ 24 August 2019 \\ Available Online: \\ 10 September 2019}

\section{A B S T R A C T}

An $8 \times 8$ diallel cross mating design with the parents and $F_{1 s}$ was used to estimate gene action and combining ability for yield and yield attributing traits in sesame (Sesamum indicum L.). Combing ability analysis revealed predominance of nonadditive gene action for all traits viz,. days $50 \%$ flowering, days to maturity, plant height, number of branches per plant, number of capsules per plant, length of capsule, width of capsule, 1000 seed weight, yield per plant and oil content which can be improved by bi-parental mating or reciprocal recurrent selection. The parent AKT-306, LOCAL and G-1 were found as good combiner for seed yield per plant. The highest SCA effect for seed yield per plant was exhibited by cross G-1 x IC- 204025 along with superior SCA effect for five characters like plant height, number capsule per plant, seed per capsule, capsule length and capsule width. The character test weight had fixable additive genetic variance which can improve by simple selection. The crosses AKT-306 x YLM-17 and LOCAL x AKT-101 with significant SCA effects indicating the predominance of additive gene action for seed yield hence progeny selection in the segregating generation is more useful.

\section{Introduction}

Sesame (Sesamum indium L.) is an ancient oil yielding crop and popularly known as "Queen of oil seeds. Worldwide, it is used for its nutritional, medicinal, and industrial purposes. Sesame is commonly known as Til (Hindi/Marathi), Tai (Gujrat), Tili (Punjab), Nuvulu (telgu), Ellu (Tamil), Rasi (Orissa) besides these sesamum, gingelly, benniseed, simsim. The crop has its early origins in East Africa and India (Bedigian and Harlan 1986). Today, India and China is the world's largest producer of sesamum followed by Myanmar, Sudan, Uganda, Nigeria, Pakistan, Tanzania, Ethopia and Turkey. Sesame seed (approximately 50\% oil, 25\% protein and 15\% carbohydrates) reported by (Burden 2005) are used in baking, candy making and other food industries. 


\section{Materials and Methods}

The experimental material for the present study comprised of eight parent viz., LOCAL, G-1, AKT-306, AKT-101, IC-205283, IC203871, IC-204025 and YLM-17.Crosses were made among the parents in diallel fashion without reciprocals during, summer 2018. The resulting $28 \mathrm{~F}_{1} \mathrm{~s}$, their parents and standard check JIT-408 and phule til -1 were sown with a spacing of $45 \mathrm{~cm}$ between rows and $20 \mathrm{~cm}$ between plants during kharif, 2018 at research farm of agriculture botany, college of agriculture, Latur. The experiment was laid out in randomized block design with three replications Five plant were selected randomly from each replication and observation were recorded for various quantitative traits viz., $50 \%$ flowering, Plant height $(\mathrm{cm})$, number of branches per plant, number of capsules per plant, Length of capsule $(\mathrm{cm})$, width of capsule (mm), test weight (gm), seed yield per plant (gm), oil content. The mean data was analysed by following method given by Griffings (1956).

\section{Results and Discussion}

Analysis of variance for combining ability indicated significant differences among treatment for all the traits. The data pertaining to the analysis of variance (Table-2) revealed significant differences among treatment, parents and $F_{1} s$ for all the characters (except days to maturity and width of capsule for Parent $v / s \mathrm{~F}_{1} \mathrm{~s}$ ) indicated the presence of substantial amount of genetic variability among the treatments, parents and crosses in the experimental material used. The estimates of components of variance revealed different types of gene action for the characters studied. Dominant gene action was found to be more pronounced for the expression all traits (Table-1). Seed yield forms the major objective in any plant breeding programme and SCA variance was more than GCA variance exhibiting the role of non-additive gene action for inheritance of this trait. The character seed yield per plant is governed by both additive and non-additive gene action. non-additive gene action was preponderant over additive in the inheritance of seed yield per plant. Thus non additive gene action could be exploited by heterosis breeding. Goyal et al., (1991), Thiyagu et al., (2007), Parimala et al., (2015) and Chudhari et al., (2015) also reported the role of non- additive gene action for seed yield. However, importance of both additive and non-additive gene action was recorded by Kadu et al., (1992).The results of GCA effects indicated that the parents, AKT306, G-1 and LOCAL were proved as good general combiner for nine, six and five character including seed yield per plant and their contributing character. Parent IC203871 and IC-204025 registered good general combiner for oil content but poor general combiner seed yield per plant. Parent IC-205283 exhibited good general combiner in earliness for characters days to $50 \%$ flowering and days to maturity. The GCA effects of parents were positively and significant associated with their mean value for majority of characters, However, these not true for all characters in all case, suggesting that inert allelic interaction were important in for these complementary epistatics effects. A close relationship between parent per se performance and their GCA effects is important in the choice of parents for crossing programme.

Good general combining ability of AKT-306 and LOCAL for one or more yield attributes might have resulted into high combining ability of these parent for seed yield these parent could therefore be effectively utilized in breeding programme for developing high yielding varieties/hybrids. The same results on general combining ability for these traits were reported previously by Khorgade et al., (1989). 


\section{Int.J.Curr.Microbiol.App.Sci (2019) 8(9): 2747- 2754}

Table.1 Analysis of variance for different characters in Sesame

\begin{tabular}{|c|c|c|c|c|c|c|c|c|c|c|c|c|c|c|c|c|c|c|c|c|c|c|c|}
\hline \multirow{2}{*}{$\begin{array}{l}\text { Source } \\
\text { Replications }\end{array}$} & \multirow{2}{*}{$\begin{array}{l}\text { D.F. } \\
2\end{array}$} & \multicolumn{2}{|c|}{$\begin{array}{l}\text { Days to } 50 \\
\text { per cent } \\
\text { flowering }\end{array}$} & \multicolumn{2}{|c|}{$\begin{array}{l}\text { Days to } \\
\text { maturity }\end{array}$} & \multicolumn{2}{|c|}{$\begin{array}{l}\text { Plant } \\
\text { height }(\mathrm{cm})\end{array}$} & \multicolumn{2}{|c|}{$\begin{array}{l}\text { Number of } \\
\text { branches }\end{array}$} & \multicolumn{2}{|c|}{$\begin{array}{l}\text { Number of } \\
\text { capsule per } \\
\text { plant }\end{array}$} & \multicolumn{2}{|c|}{$\begin{array}{lr}\begin{array}{l}\text { Number } \\
\text { seed }\end{array} & \text { pe } \\
\text { capsule } & \end{array}$} & \multicolumn{2}{|c|}{$\begin{array}{l}\text { Length of } \\
\text { capsule } \\
\text { (cm) }\end{array}$} & \multicolumn{2}{|c|}{$\begin{array}{l}\text { Width of } \\
\text { capsule } \\
(\mathrm{mm})\end{array}$} & \multicolumn{2}{|c|}{$\begin{array}{l}1000 \text { Seed } \\
\text { weight }(g)\end{array}$} & \multicolumn{2}{|c|}{$\begin{array}{ll}\text { Seed } & \text { yield } \\
\text { per } & \text { plant } \\
(\mathrm{g}) & \end{array}$} & \multicolumn{2}{|c|}{$\begin{array}{l}\text { Oil content } \\
(\%)\end{array}$} \\
\hline & & 2.583 & & 3.814 & & 20.721 & & 0.107 & & 10.015 & & 1.605 & & 0.054 & & 0.006 & & 0.005 & & 0.945 & & 3.374 & \\
\hline Treatments & 35 & 29.180 & $* *$ & 12.733 & $* *$ & 667.54 & $* *$ & 2.426 & $* *$ & 529.65 & * & 126.95 & $* *$ & 0.210 & * & 1.165 & * & 0.930 & * & 44.044 & $\begin{array}{l}* \\
*\end{array}$ & 45.779 & $* *$ \\
\hline Parents & 7 & 22.375 & $* *$ & 3.184 & $*$ & 288.36 & $* *$ & 0.933 & $* *$ & 332.35 & * & 31.29 & $* *$ & 0.208 & * & 0.303 & $\begin{array}{l}* \\
*\end{array}$ & 0.685 & * & 18.128 & $\begin{array}{l}* \\
*\end{array}$ & 38.560 & $* *$ \\
\hline$F_{1}^{\prime} s$ & 27 & 31.786 & $* *$ & 15.679 & $* *$ & 764.25 & $* *$ & 2.796 & $* *$ & 576.28 & * & 133.78 & $* *$ & 0.192 & $\begin{array}{l}* \\
*\end{array}$ & 1.432 & $\begin{array}{l}* \\
*\end{array}$ & 0.991 & * & 52.051 & $\begin{array}{l}* \\
*\end{array}$ & 48.699 & $* *$ \\
\hline $\begin{array}{l}\text { Parents v/s } \\
F_{1} \text { 's }\end{array}$ & 1 & 6.482 & $*$ & 0.032 & & 710.50 & $* *$ & 2.880 & $* *$ & 651.78 & * & 613.46 & $* *$ & 0.718 & * & 0.001 & & 0.995 & * & 9.271 & * & 17.468 & $* *$ \\
\hline Error & 70 & 1.535 & & 1.252 & & 44.397 & & 0.230 & & 10.508 & & 5.423 & & 0.022 & & 0.020 & & 0.013 & & 0.912 & & 1.451 & \\
\hline GCA & 7 & 33.098 & $* *$ & 7.767 & $* *$ & 503.66 & $* *$ & 1.070 & $* *$ & 288.770 & * & 42.545 & $* *$ & 0.120 & * & 0.927 & * & 0.464 & * & 36.396 & $\begin{array}{l}* \\
*\end{array}$ & 33.830 & $* *$ \\
\hline SCA & 28 & 3.884 & $* *$ & 3.363 & $* *$ & 152.22 & $* *$ & 0.743 & $* *$ & 148.497 & $\begin{array}{l}* \\
*\end{array}$ & 42.277 & $* *$ & 0.057 & * & 0.253 & $\begin{array}{l}* \\
*\end{array}$ & 0.271 & * & 9.252 & $\begin{array}{l}* \\
*\end{array}$ & 10.617 & $* *$ \\
\hline$\delta 2 \mathrm{gca} / \mathrm{sca}$ & & 0.8663 & & 0.1494 & & 0.2557 & & 0.0491 & & 0.0967 & & 0.0006 & & 0.1246 & & 0.2728 & & 0.0722 & & 0.3033 & & 0.2290 & \\
\hline
\end{tabular}


Table.2 Estimates of general and specific combining ability effect for different characters in Sesame

\begin{tabular}{|c|c|c|c|c|c|c|c|c|c|}
\hline \multirow[t]{2}{*}{$\begin{array}{l}\text { Sr. } \\
\text { No. }\end{array}$} & \multirow{2}{*}{$\begin{array}{l}\text { Parents/ crosses } \\
\text { Parents }\end{array}$} & \multicolumn{2}{|c|}{$\begin{array}{l}\text { Days to } 50 \% \\
\text { flowering }\end{array}$} & \multicolumn{2}{|c|}{$\begin{array}{ll}\text { Days } & \text { to } \\
\text { maturity } & \end{array}$} & \multicolumn{2}{|c|}{ Plant height } & \multicolumn{2}{|c|}{ No. of branches } \\
\hline & & & & & & & & & \\
\hline 1 & LOCAL & 1.000 & $* *$ & 0.142 & & -1.979 & & 0.535 & $* *$ \\
\hline 2 & G-1 & -1.267 & $* *$ & -0.658 & $* *$ & -10.092 & $* *$ & 0.255 & $* *$ \\
\hline 3 & AKT-306 & -0.800 & $* *$ & 0.275 & & 4.110 & $* *$ & 0.222 & $* *$ \\
\hline 4 & AKT-101 & 1.833 & $* *$ & 0.808 & $* *$ & -0.145 & & -0.225 & $* *$ \\
\hline 5 & IC-205283 & -3.200 & $* *$ & -1.225 & $* *$ & -9.912 & $* *$ & -0.378 & $* *$ \\
\hline 6 & IC-203871 & 0.633 & $* *$ & -0.058 & & 9.577 & $* *$ & -0.092 & $* *$ \\
\hline 7 & IC-204025 & -0.576 & $* *$ & -0.758 & $* *$ & 5.684 & $* *$ & -0.378 & $* *$ \\
\hline \multirow[t]{4}{*}{8} & YLM-17 & 2.367 & $* *$ & 1.475 & $* *$ & 2.757 & $*$ & 0.062 & \\
\hline & S.E.gi & 0.2116 & & 0.1911 & & 1.137 & & 0.0821 & \\
\hline & S.E.gi-gi & 0.3199 & & 0.2890 & & 1.720 & & 0.1241 & \\
\hline & \multicolumn{9}{|l|}{ Crosses } \\
\hline 9 & LOCAL x G-1 & 1.933 & $* *$ & 1.193 & & 16.237 & $* *$ & 0.671 & $*$ \\
\hline 10 & LOCAL x AKT-306 & -3.867 & $* *$ & -1.741 & $* *$ & 1.458 & & 0.904 & $*$ \\
\hline 11 & LOCAL x AKT-101 & -0.500 & & 0.393 & & 0.513 & & 0.818 & $* *$ \\
\hline 12 & LOCAL x IC-205283 & -0.467 & & -0.241 & & -26.120 & $* *$ & -1.096 & $* *$ \\
\hline 13 & LOCAL x IC-203871 & 2.700 & $* *$ & 1.259 & $*$ & 7.924 & $*$ & 0.684 & $* *$ \\
\hline 14 & LOCAL x IC-204025 & 3.567 & $* *$ & 1.626 & $* *$ & 1.317 & & 0.771 & $* *$ \\
\hline 15 & LOCAL x YLM-17 & -2.033 & $* *$ & -2.607 & $* *$ & 1.644 & & -0.202 & \\
\hline 16 & G-1 x AKT-306 & -2.267 & $* *$ & -2.941 & $* *$ & 17.071 & $* *$ & 1.051 & $* *$ \\
\hline 17 & G-1 x AKT-101 & 0.433 & & 2.526 & $* *$ & -15.707 & $* *$ & 0.031 & \\
\hline 18 & G-1 x IC-205283 & -1.533 & $*$ & -1.107 & & -21.774 & $* *$ & -1.482 & $* *$ \\
\hline 19 & G-1 x IC-203871 & 0.633 & & 0.059 & & 7.037 & & 0.431 & \\
\hline 20 & G-1 x IC-204025 & -0.833 & & 0.426 & & 16.664 & $* *$ & 0.784 & $* *$ \\
\hline 21 & G-1 x YLM-17 & 1.233 & & -0.807 & & -11.076 & $* *$ & -0.989 & $* *$ \\
\hline 22 & AKT-306 x AKT-101 & -0.033 & & -0.407 & & -3.043 & & -0.784 & $* *$ \\
\hline 23 & AKT-306 x IC-205283 & 1.667 & $*$ & 0.293 & & 2.291 & & 1.084 & $* *$ \\
\hline 24 & AKT-306 x IC-203871 & 0.500 & & 2.793 & $* *$ & 1.901 & & 0.931 & $* *$ \\
\hline 25 & AKT-306 x IC-204025 & -2.300 & $* *$ & -1.507 & $*$ & -4.072 & & -0.849 & $* *$ \\
\hline 26 & AKT-306 x YLM-17 & 1.767 & $*$ & 3.926 & $* *$ & 7.122 & & -0.156 & \\
\hline 27 & AKT-101 x IC-205283 & 2.033 & $* *$ & 2.093 & $* *$ & 0.226 & & 0.531 & $*$ \\
\hline 28 & AKT-101 x IC-203871 & -0.467 & & -0.074 & & -3.843 & & -0.356 & \\
\hline 29 & AKT-101 x IC-204025 & -1.600 & $*$ & -2.041 & $* *$ & -3.883 & & -0.536 & $*$ \\
\hline 30 & AKT-101 x YLM-17 & 0.133 & & 1.393 & & 17.311 & $* *$ & -0.509 & \\
\hline 31 & IC-205283 x IC-203871 & -3.100 & $* *$ & -2.041 & $* *$ & 18.157 & $* *$ & 0.464 & \\
\hline 32 & IC-205283 x IC-204025 & -2.900 & $* *$ & -2.341 & $* *$ & 5.950 & & -0.782 & $* *$ \\
\hline 33 & IC-205283 x YLM-17 & 0.833 & & 1.759 & $* *$ & 4.844 & & -0.089 & \\
\hline 34 & IC-203871 x IC-204025 & -0.733 & & -0.507 & & 2.767 & & -0.469 & \\
\hline 35 & IC-203871 x YLM-17 & 1.00 & & -1.074 & & 8.588 & * & 0.624 & * \\
\hline 36 & IC-204025 x YLM-17 & 0.533 & & -0.041 & & -11.119 & $* *$ & 0.911 & $* *$ \\
\hline & S.E sij & 0.6487 & & 0.5860 & & 3.488 & & 0.2517 & \\
\hline & S.E sij - sik & 0.9599 & & 0.8670 & & 5.161 & & 0.3724 & \\
\hline
\end{tabular}

* Significant at $5 \%$ level, ** Significant at $1 \%$ level 
Int.J.Curr.Microbiol.App.Sci (2019) 8(9): 2747- 2754

Table.3 Estimates of general and specific combining ability effect for different characters in Sesame

\begin{tabular}{|c|c|c|c|c|c|c|c|c|c|}
\hline \multirow[t]{2}{*}{$\begin{array}{l}\text { Sr. } \\
\text { No. }\end{array}$} & \multirow{2}{*}{$\begin{array}{l}\text { Parents/ crosses } \\
\text { Parents }\end{array}$} & \multicolumn{2}{|c|}{$\begin{array}{l}\text { No of capsule } \\
\text { per plant }\end{array}$} & \multicolumn{2}{|c|}{$\begin{array}{l}\text { No of seeds per } \\
\text { capsule }\end{array}$} & \multicolumn{2}{|c|}{$\begin{array}{l}\text { Capsule length } \\
(\mathrm{cm})\end{array}$} & \multicolumn{2}{|c|}{$\begin{array}{l}\text { Capsule width } \\
(\mathrm{mm})\end{array}$} \\
\hline & & & & & & & & & \\
\hline 1 & LOCAL & 4.121 & $* *$ & -0.528 & & 0.053 & $*$ & 0.583 & $* *$ \\
\hline 2 & G-1 & -0.349 & & -1.694 & $* *$ & 0.070 & $* *$ & 0.259 & $* *$ \\
\hline 3 & AKT-306 & 10.689 & $* *$ & 3.626 & $* *$ & 0.190 & $* *$ & 0.118 & $* *$ \\
\hline 4 & AKT-101 & -2.847 & $* *$ & -1.601 & $* *$ & 0.001 & & -0.123 & $* *$ \\
\hline 5 & IC-205283 & -7.511 & $* *$ & 1.113 & $* *$ & 0.017 & & -0.353 & $* *$ \\
\hline 6 & IC-203871 & -1.628 & $* *$ & 0.859 & $*$ & -0.086 & $* *$ & -0.214 & $* *$ \\
\hline 7 & IC-204025 & -0.714 & & 1.033 & $*$ & -0.159 & $* *$ & -0.078 & $* *$ \\
\hline \multirow[t]{3}{*}{8} & YLM-17 & -1.761 & $* *$ & -2.808 & $* *$ & -0.085 & $* *$ & -0.192 & $* *$ \\
\hline & S.E.gi & 0.5536 & & 0.3977 & & 0.02540 & & 0.0243 & \\
\hline & S.E.gi-gi & 0.8370 & & 0.6012 & & 0.03841 & & 0.0368 & \\
\hline \multicolumn{10}{|c|}{ Crosses } \\
\hline 9 & LOCAL x G-1 & 5.415 & $* *$ & -0.016 & & 0.63 & & 0.165 & $*$ \\
\hline 10 & LOCAL x AKT-306 & 3.877 & $*$ & 5.930 & $* *$ & 0.209 & $*$ & 0.350 & $* *$ \\
\hline 11 & LOCAL x AKT-101 & 20.786 & $* *$ & 3.090 & $*$ & 0.332 & $* *$ & 0.418 & $* *$ \\
\hline 12 & LOCAL x IC-205283 & -4.003 & $*$ & -7.623 & $* *$ & -0.181 & $*$ & -0.359 & $* *$ \\
\hline 13 & LOCAL x IC-203871 & -0.806 & & -1.503 & & -0.001 & & 0.508 & $* *$ \\
\hline 14 & LOCAL x IC-204025 & 1.114 & & -2.476 & & 0.172 & $*$ & -0.057 & \\
\hline 15 & LOCAL x YLM-17 & -6.339 & $* *$ & 1.097 & & 0.172 & $*$ & 0.470 & $* *$ \\
\hline 16 & G-1 x AKT-306 & 5.867 & $* *$ & -2.836 & $*$ & 0.393 & $* *$ & 0.366 & $* *$ \\
\hline 17 & G-1 x AKT-101 & -5.217 & $* *$ & 2.124 & & -0.251 & $* *$ & 0.141 & \\
\hline 18 & G-1 x IC-205283 & -9.220 & $* *$ & 1.010 & & -0.090 & & 0.064 & \\
\hline 19 & G-1 x IC-203871 & 4.831 & $* *$ & -0.336 & & 0.103 & & -0.315 & $* *$ \\
\hline 20 & G-1 x IC-204025 & 24.221 & $* *$ & 12.557 & $* *$ & 0.572 & $* *$ & 0.863 & $* *$ \\
\hline 21 & G-1 x YLM-17 & 2.064 & & 0.664 & & -0.251 & $* *$ & 0.210 & $* *$ \\
\hline 22 & AKT-306 x AKT-101 & 3.898 & $*$ & -2.130 & & 0.195 & $*$ & -0.434 & $* *$ \\
\hline 23 & AKT-306 x IC-205283 & 6.896 & $* *$ & -0.576 & & -0.088 & & -0.198 & $*$ \\
\hline 24 & AKT-306 x IC-203871 & 6.837 & $* *$ & 5.544 & $* *$ & -0.058 & & -0.354 & $* *$ \\
\hline 25 & AKT-306 x IC-204025 & -2.487 & & 5.904 & $* *$ & -0.118 & & -0.330 & $* *$ \\
\hline 26 & AKT-306 x YLM-17 & 14.293 & $* *$ & 8.744 & $* *$ & 0.215 & $*$ & 0.918 & $* *$ \\
\hline 27 & AKT-101 x IC-205283 & 14.645 & $* *$ & 1.984 & & 0.035 & & 0.210 & $* *$ \\
\hline 28 & AKT-101 x IC-203871 & 11.849 & $* *$ & -0.696 & & -0.068 & & 0.148 & \\
\hline 29 & AKT-101 x IC-204025 & -16.152 & $* *$ & 7.130 & $* *$ & -0.149 & & -0.938 & $* *$ \\
\hline 30 & AKT-101 x YLM-17 & -9.171 & $* *$ & -7.563 & $* *$ & -0.129 & & -0.180 & $* *$ \\
\hline 31 & IC-205283 x IC-203871 & 11.226 & $* *$ & 4.057 & $* *$ & -0.188 & $* *$ & -0.056 & \\
\hline 32 & IC-205283 x IC-204025 & -11.488 & $* *$ & -0.650 & & 0.048 & & 0.338 & $* *$ \\
\hline 33 & IC-205283 x YLM-17 & -4.860 & $* *$ & 12.257 & $* *$ & 0.078 & & -0.481 & $* *$ \\
\hline 34 & IC-203871 x IC-204025 & -21.303 & $* *$ & 6.604 & $* *$ & 0.095 & & -0.398 & $* *$ \\
\hline 35 & IC-203871 x YLM-17 & -8.790 & $* *$ & -7.356 & $* *$ & 0.045 & & -0.503 & $* *$ \\
\hline \multirow[t]{3}{*}{36} & IC-204025 x YLM-17 & -1.270 & & -9.263 & $* *$ & 0.064 & & -0.519 & $* *$ \\
\hline & S.E sij & 1.6971 & & 1.2191 & & 0.0778 & & 0.0368 & \\
\hline & S.E sij - sik & 2.5110 & & 1.8038 & & 0.1152 & & 0.1103 & \\
\hline
\end{tabular}

* Significant at $5 \%$ level, ** Significant at $1 \%$ level 
Table.4 Estimates of general and specific combining ability effect for different characters in sesame

\begin{tabular}{|c|c|c|c|c|c|c|c|}
\hline \multirow[t]{2}{*}{$\begin{array}{l}\text { Sr. } \\
\text { No. }\end{array}$} & \multirow{2}{*}{$\begin{array}{l}\text { Parents/ crosses } \\
\text { Parents }\end{array}$} & \multicolumn{2}{|c|}{$\begin{array}{l}1000 \text { seed weight } \\
\text { (g) }\end{array}$} & \multicolumn{2}{|c|}{$\begin{array}{l}\text { Seed yield per } \\
\text { plant }(\mathrm{g})\end{array}$} & \multicolumn{2}{|c|}{ Oil content (\%) } \\
\hline & & & & & & & \\
\hline 1 & LOCAL & 0.177 & $* *$ & 1.443 & $* *$ & -0.850 & ** \\
\hline 2 & G-1 & -0.063 & $* *$ & 0.395 & $*$ & -2.864 & $* *$ \\
\hline 3 & AKT-306 & 0.337 & $* *$ & 3.811 & $* *$ & 2.118 & $* *$ \\
\hline 4 & AKT-101 & -0.336 & $* *$ & -0.997 & $* *$ & -0.138 & \\
\hline 5 & IC-205283 & -0.053 & $* *$ & -1.940 & $* *$ & -0.373 & \\
\hline 6 & IC-203871 & 0.130 & $* *$ & -0.146 & & 0.630 & $* *$ \\
\hline 7 & IC-204025 & 0.015 & & -0.713 & $* *$ & 2.815 & $* *$ \\
\hline \multirow[t]{3}{*}{8} & YLM-17 & -0.208 & $* *$ & -1.852 & $* *$ & -1.339 & $* *$ \\
\hline & S.E.gi & 0.0195 & & 0.1631 & & 0.2057 & \\
\hline & S.E.gi-gi & 0.0296 & & 0.2465 & & 0.3110 & \\
\hline \multicolumn{8}{|c|}{ Crosses } \\
\hline 9 & LOCAL x G-1 & 0.260 & $* *$ & -0.581 & $* *$ & 1.319 & $*$ \\
\hline 10 & LOCAL x AKT-306 & 0.127 & $*$ & 1.120 & $*$ & 2.377 & $* *$ \\
\hline 11 & LOCAL x AKT-101 & -0.300 & $* *$ & 4.681 & $* *$ & -4.857 & $* *$ \\
\hline 12 & LOCAL x IC-205283 & -0.416 & $* *$ & -3.863 & ** & -1.365 & $*$ \\
\hline 13 & LOCAL x IC-203871 & 0.400 & $* *$ & -0.024 & & 0.169 & \\
\hline 14 & LOCAL x IC-204025 & -0.449 & $* *$ & -1.026 & $*$ & 4.653 & $* *$ \\
\hline 15 & LOCAL x YLM-17 & -0.561 & $*$ & 3.739 & $* *$ & 1.004 & \\
\hline 16 & G-1 x AKT-306 & 0.434 & $* *$ & 0.452 & & -0.639 & \\
\hline 17 & G-1 x AKT-101 & -0.526 & $* *$ & -1.458 & $* *$ & -2.029 & $* *$ \\
\hline 18 & G-1 x IC-205283 & 0.157 & $*$ & 0.269 & & 4.769 & $* *$ \\
\hline 19 & G-1 x IC-203871 & -0.193 & $* *$ & 2.508 & $* *$ & -0.994 & \\
\hline 20 & G-1 x IC-204025 & 0.422 & $* *$ & 7.176 & $* *$ & 2.448 & $* *$ \\
\hline 21 & G-1 x YLM-17 & 0.512 & $* *$ & -1.586 & $* *$ & -7.089 & $* *$ \\
\hline 22 & AKT-306 x AKT-101 & -0.626 & $* *$ & 1.187 & $*$ & -3.891 & $* *$ \\
\hline 23 & AKT-306 x IC-205283 & 0.324 & $* *$ & 1.583 & $* *$ & 4.417 & $* *$ \\
\hline 24 & AKT-306 x IC-203871 & 0.107 & & 0.659 & & 1.354 & $*$ \\
\hline 25 & AKT-306 x IC-204025 & -0.145 & $*$ & 0.290 & & -0.198 & \\
\hline 26 & AKT-306 x YLM-17 & 0.445 & $* *$ & 4.545 & $* *$ & -0.421 & \\
\hline 27 & AKT-101 x IC-205283 & 0.197 & $* *$ & -0.523 & & 2.664 & $* *$ \\
\hline 28 & AKT-101 x IC-203871 & 0.980 & $* *$ & 2.563 & $* *$ & 0.757 & \\
\hline 29 & AKT-101 x IC-204025 & -0.671 & $* *$ & -2.743 & $* *$ & 1.622 & $*$ \\
\hline 30 & AKT-101 x YLM-17 & 0.719 & $* *$ & -0.987 & & 1.589 & $*$ \\
\hline 31 & IC-205283 x IC-203871 & 0.097 & & -2.720 & $* *$ & -3.328 & $* *$ \\
\hline 32 & IC-205283 x IC-204025 & -0.021 & & -1.243 & $*$ & -4.470 & $* *$ \\
\hline 33 & IC-205283 x YLM-17 & -1.165 & $* *$ & -0.457 & & -2.916 & $* *$ \\
\hline 34 & IC-203871 x IC-204025 & -0.305 & $* *$ & -4.070 & ** & -5.413 & $* *$ \\
\hline 35 & IC-203871 x YLM-17 & 0.285 & $* *$ & -3.585 & $* *$ & -0.106 & \\
\hline \multirow[t]{3}{*}{36} & IC-204025 x YLM-17 & 0.450 & $* *$ & -1.517 & $* *$ & 2.539 & *** \\
\hline & S.E sij & 0.0600 & & 0.4999 & & 0.6306 & \\
\hline & S.E sij-sik & 0.0888 & & 0.7397 & & 0.9331 & \\
\hline
\end{tabular}

* Significant at $5 \%$ level, ** Significant at $1 \%$ level 
Among the crosses studied, G-1 x IC-204025, AKT-306 x YLM-17, LOCAL x AKT-306 and LOCAL $x$ AKT-101 were identified as the potential specific combiners for seed yield per plant and other yield contributing traits (Table-2,3,4).Highly significant positive SCA effect for number of capsule per plant was exhibiting in the crosses G-1 x IC-204025 (24.221), LOCAL x AKT-101 (20.786) and AKT-101 x IC-205283 (14.645). The hybrid G-1 x IC-204025, IC-205283 x YLM-17 and AKT-306 x YLM-17 were found to be good specific combiners for number of seed per capsules. The crosses, AKT-306 x IC-205283, G-1 x AKT-306 and AKT-306 x IC-203871 showed highly significant positive SCA effect for number of branches per plant. The crosses AKT-101 x IC-203871, AKT-101 x YLM-17 and G-1 x YLM-17 exhibited highest positive significant SCA effect for test weight. Good specific combiners for oil content were G-1 X IC-205283, LOCAL x IC-204025 and AKT$306 \times$ IC-205283. The cross LOCAL x AKT306 had both good combining parent for seed yield. Raikwar (2018) reported close relationship between GCA effects of parents and SCA effects of their resultant crosses. The cross, G-1 x IC-204025 had good x poor combining parents. Positive SCA in crosses between good and poor combiners could be ascribed to better complementation between favorable alleles of the parents involved. Goyal and kumar (1986) and Rajput and Kute (2017) also stressed the importance of good $\mathrm{x}$ poor crosses in obtaining superior combination.

The highest SCA effect for seed yield per plant was exhibited by cross G-1 x IC- 204025 along with superior SCA effect for five characters like plant height, number capsule per plant, seed per capsule, capsule length and capsule width. The character test weight had fixable additive genetic variance which can improve by simple selection. The crosses AKT-306 x YLM-17 and LOCAL x AKT-101 with significant SCA effects indicating the predomince of additive gene action for seed yield hence progeny selection in the segregating generation is more useful.

\section{References}

Bedigian, D. and Harlan, J. R., (1986). Evidence for cultivation of sesame in the ancient world. Economic Botany, 40, 137-154.

Burden, D., (2005). Sesame profile. Mhtml:file://C:IDocuments and settingsluse rllDesktoplcrop profiles.mht (15/01/08).

Chaudhari, G. B., Naik, M. R., Anarase, S. A. and Ban, Y. G., (2015a).Heterosis studies for quantitative traits in sesame (Sesamum indicum L.). Electronic J. Plt. Breed., 6(1): 218-224.

Goyal, S. N and Kumar, S. (1986). Heterosis in relation to general and specific combining ability in sesame (Sesamum indicum L.). Ind. J. Genet., 48(2): 251253.

Goyal, S. N. and Kumar, S. (1991a). Combining ability for yield components and oil content in sesame (Sesamum indicum L.). Ind. J. Genet., 51(3): 311-314.

*Griffings, B. (1956) (a). Concept of general and specific combining ability relation to diallel crossing system. Aust. J. Bil. Sci., 9:463-493.

Kadu, S., Narkhede, M. N. and Khorgade, P. W. 1992. Studies on combining ability in sesame. J. Maharashtra Agric. Univ., 17(3): 392 - 393.

Khorgade, P. W., Deshmukh, A. V., Narkhede, M. N. and Raut, S. K. (1989). Combining ability for yield and its components in sesame. $J$. Maharashtra Agric. Univ., 14(2): 164 166.

Parimala, K., Reddy, V. A. and Rao, P.V.R., (2015a). Exploitation of hybrid vigour 
in sesame (Sesamum indicum L.). Electronic J. of Plt. Breed., 6(1): 125129.

Raikwar, R. S. (2018). Diallel crossing for assessment of yield and its components in sesame (Sesamum indicum L.). Int. J. of Chemical Studies., 6(2): 179-183.

Rajput, S. D. and Kute, N. S. (2017). Gene effects for yield and fatty acids in sesame. Int J. of Current Res., 9(09): 56966-56970.

Thiyagu, K., Kandasamy, G., Manivannan, N. and Muralidharan, V. (2007a). Studies on heterosis in genetically diverse lines of cultivated sesame (Sesamum indicum L.) Madras Agric. J., 94(712): $162-167$.

\section{How to cite this article:}

Sonawane, S. J., P. B. Wadikar, M. R. Magar and Dhare, S. L. 2019. Combining Ability and Gene Action in Sesame (Sesamum indicum L.) Elite Genotypes by Half Diallel Mating Design. Int.J.Curr.Microbiol.App.Sci. 8(09): 2747- 2754.doi: https://doi.org/10.20546/ijcmas.2019.809.316 